Königs, wenn er behauptet, eine neue normative Grundlage für den Menschenrechtsdiskurs innerhalb der Soziologie zu finden. Im Zuge der „metatheoretischen Suspension der Begründungsfrage“ will er diese in der Analyse der „empirischen Entstehungs- und Geltungsbedingungen" der Menschenrechte finden (154). Die exakte Begründung, wie eine „metatheoretische Suspension" aus dem Problem herausführt, dass aus einem gesellschaftlichen Sein kein moralisches Sollen postuliert werden kann, bleibt der Autor jedoch schuldig. Natürlich kann diese Verwechslung zwischen der Analyse sozialen Seins und der Begründung normativen Sollens auf eine gewisse Tradition in der Soziologie zurückblicken, eine Verwechslung, der Durkheim durchaus hin und wieder erlegen ist. Gerade die Lektüre Webers, der diese beiden Aspekte ja bekanntlich klar scheidet (was der Autor im Übrigen auch bemerkt) hätte König dazu bewegen können, diesen Aspekt ausführlicher zu diskutieren. Damit wäre das Buch zum überwiegenden Teil der Forschung zu normativen Urteilen in der Soziologie kompatibel geworden, die ja bekanntlich und vermutlich auch zu Recht auf strikter Trennung zwischen normativen Geltungsansprüchen und empirischen Geltungsansprüchen bestehen.

Da sich der größte Teil des Buches jedoch der Darstellung der Theorie von Durkheim und Weber widmet, ist diese "intellektuelle Abkürzung“ $\mathrm{zu}$ verschmerzen. Insbesondere als Einführung in die soziologische Analyse normativer Muster bei Durkheim und Weber ist das Werk auf das Beste geeignet. Gerade seine Kürze wird hier zum Vorteil, und die Klarheit von Sprache und Struktur nehmen den Lesenden für das Buch ein. Darüber hinaus hinterlässt es den Leser insofern in positiver Stimmung, als es plausibel macht, dass zwei Gründerväter der Soziologie, sei es mit funktionalen oder historischen Begründungen, in modernen Gesellschaften eine Möglichkeit zur Verwirklichung der Menschenrechte sehen.

Mathias Bös

\section{SOZIOLOGIE DER WIRTSCHAFT}

Martin Höpner: Wer beherrscht die Unternehmen? Shareholder Value, Managerherrschaft und Mitbestimmung in Deutschland. Frankfurt a.M./New York: Campus 2003. 265 Seiten. ISBN 3-593-37266-5. Preis: € 29,90.

Die Praxis aktionärsorientierter Unternehmensführung - auch unter dem Schlagwort „Sharehol- der Value“ (SV) bekannt - gilt seit den 90er Jahren auch deutschen Topmanagern als eine Selbstverständlichkeit, der sich andere Interessengruppen offenbar zu beugen haben. Welche Strategien verbergen sich hinter dem SV-Konzept? Was sind die Ursachen für die Hinwendung deutscher Unternehmen zur „kapitalmarktorientierten Unternehmensführung"? Und schließlich: Welche Folgen hat die unternehmensstrategische Neuorientierung für das System industrieller Beziehungen in Deutschland? Diesen Fragen hat sich Martin Höpner in seiner Dissertation „Wer beherrscht die Unternehmen?" gewidmet.

Theoretischer Ausgangspunkt der Untersuchung ist die von Peter A. Hall und David Soskice (weiter)entwickelte Theorie der Spielarten des Kapitalismus, der zufolge das Modell Deutschland einen paradigmatischen Fall einer koordinierten Marktökonomie darstellt, deren Teilbereiche der gleichen marktbeschränkenden Logik folgen und sich dadurch wechselseitig stabilisieren. Demnach werden ein Stakeholderorientiertes, d.h. machtverteilendes Corporate Governance-System, stabile Finanzbeziehungen zwischen Hausbanken und Industrieunternehmen sowie zentralisierte Lohnverhandlungen und Arbeitnehmermitbestimmung als komplementäre Arrangements aufgefasst, die deutschen Unternehmen aufgrund ihrer Kohärenz jahrzehntelang Wettbewerbsvorteile verschafft haben. Vor diesem Hintergrund muss der Wandel des deutschen Corporate Governance-Systems im Verlauf der 90er Jahre und die hierdurch bedingte Hinwendung deutscher Großunternehmen zu aktionärsorientierten Konzepten der Unternehmensführung als Störfaktor in der deutschen Wirtschaftsordnung erscheinen, der als solcher erklärungsbedürftig ist. Zudem wirft die Stärkung der Marktkräfte im Ordnungssegment der Corporate Governance die Frage auf, ob und inwieweit sich Komplementärinstitutionen dieser Entwicklung angleichen und hierdurch letztlich eine Konvergenz des gesamten Produktionsregimes in Richtung liberaler Marktökonomie in Gang gesetzt wird.

In methodologischer Hinsicht handelt es sich bei der Studie um eine auf das Ende der 90er Jahre bezogene Querschnittsanalyse, für die ein Index zur Erfassung der SV-Orientierung erstellt und die 40 größten börsennotierten deutschen Unternehmen in eine nach dem Ausmaß der SVOrientierung abgestuften Rangfolge gebracht wurden (44f.). Die nachfolgend dargestellten $\mathrm{Zu}$ sammenhänge wurden mittels Korrelations- und Regressionsrechnungen validiert.

Der Mittelteil des Buches beschäftigt sich mit den Ursachen der in Kapitel 2 dokumentierten 
Hinwendung deutscher Großunternehmen zu SV-Strategien. Hierbei werden sowohl externe (Kapitel 3) als auch interne Ursachen (Kapitel 4) diskutiert. Als externe Einflussfaktoren werden von Höpner die Intensivierung des Wettbewerbs auf den Gütermärkten (79-93), der vergrößerte Einfluss der institutionellen Investoren (93-102) und die Entstehung eines Marktes für feindliche Übernahmen (104-114) hervorgehoben. Bei diesen Einflussgrößen handelt es sich um Überwachungsmechanismen, die traditionell für das angloamerikanische Corporate Governance-System kennzeichnend sind und im Verlauf der 90er Jahre in Deutschland an Bedeutung gewonnen haben. Die häufig geäußerte Behauptung, dass sich deutsche Großunternehmen aufgrund einer Verknappung der Eigenkapitalversorgung zur Implementierung kapitalmarktorientierter Unternehmensstrategien gezwungen gesehen haben, wird hingegen mit dem Hinweis auf die gleich bleibend große Bedeutung der Innenfinanzierung sowie die Absenkung der Eigenkapitalquoten bei SV-Unternehmen zurückgewiesen (115-120).

Wie der Autor im vierten Kapitel zeigt, ist die Entfaltung der externen Antriebskräfte nicht etwa auf Widerstand bei deutschen Managern getroffen, sondern "intern auf fruchtbaren Boden“" (121) gefallen. Die Bereitschaft deutscher Topmanager, sich an die Spitze der SV-Bewegung zu stellen, erklärt Höpner durch den Wandel der Führungskräfterekrutierung (123-133), den Rückzug der Banken aus der Unternehmensüberwachung und die Neubesetzung der Aufsichtsräte (133-139) sowie die hierdurch ermöglichten $\mathrm{Er}$ höhungen der Managervergütungen (139-149).

Im fünften Kapitel des Buches geht der Autor der Frage nach, inwieweit sich die Institutionen der Arbeitsbeziehungen infolge der Transformation des deutschen Corporate Governance-Regimes verändert haben. Entgegen der aus der Komplementaritätsannahme deduzierten Destabilisierungshypothese kommt Höpner zu dem Ergebnis, dass kapitalmarktorientierte Unternehmensführung keineswegs eine Erosion von Mitbestimmung und Flächentarifverträgen nach sich zieht, sondern mit diesen in Einklang gebracht werden kann. Die Kompatibilität von Aktionärsorientierung und industriellen Bürgerrechten wird in dreifacher Weise aufgezeigt: 1. Zunächst wird anhand von Umfrageergebnissen die mehrheitliche Akzeptanz der Mitbestimmung bei den Arbeitgebern aufgewiesen. Diese ist unabhängig vom Ausmaß der SV-Orientierung. Der Flächentarifvertrag wird von kapitalmarktorientierten Unternehmen nicht in Frage gestellt, da dieser den Großunternehmen als Schutz vor höheren Lohnforderungen dient. Auch auf das Urteil der
Kapitalmarktteilnehmer scheint Arbeitnehmermitbestimmung keinen negativen Einfluss auszuüben (161). 2. Sodann zeigt der Autor anhand der fehlenden Korrelation zwischen Mitbestimmungsstärke und SV-Orientierung (163), dass die Mitbestimmungsträger keinen bremsenden Einfluss auf die Implementierung des SV-Konzeptes ausgeübt haben. Differenziert man dieses nach Dimensionen, lassen sich jeweils unterschiedliche Konfliktlinien und Koalitionen aus Managern, Aktionären und Arbeitnehmern identifizieren. So stehen sich in der kommunikativen Dimension (z.B. bei Transparenzfragen) sowie in der Dimension der Managervergütung Aktionäre und Arbeitnehmer auf der einen Seite und Manager auf der anderen Seite gegenüber. In der operativen Dimension hingegen können etwa bei der Einführung von Renditevorgaben auch „Klassenkonflikte" (152) oder bei Restrukturierungswünschen der Aktionäre „Insider/Outsider-Konflikte" (ebd.) entstehen. 3. Schließlich verweist Höpner einschränkend auf die Verschlechterung der Verteilungsposition der Arbeitnehmer gegenüber den Managern und Aktionären sowie den unter dem Schlagwort "Co-Management" diskutierten Funktionswandel der Mitbestimmung. Nicht zuletzt die in SV-Unternehmen übliche Verkopplung von Vergütungsstrukturen und $\mathrm{Fi}$ nanzkennziffern in Form von Beteiligungsprogrammen hat dem Autor zufolge dazu beigetragen, die Mitarbeiter auf einzelbetriebliche Erfolge $\mathrm{zu}$ verpflichten und dadurch eine Partikularisierung von Arbeitnehmerinteressen hervorzurufen. Die „wechselseitige Durchdringung von Kapital und Arbeit" (185) wird somit von einer Tendenz zur „Mikro-Orientierung“ sowie einer hierdurch bedingten Entsolidarisierung innerhalb der Konzernbelegschaft begleitet. Bemerkenswert ist, dass Höpner in diesem Zusammenhang nicht nur die Probleme von SV, sondern auch die mit Kapitalbeteiligungsprogrammen und Pensionsfonds verbundenen Chancen der Mitbestimmung thematisiert.

Der Schlussteil enthält nach einer konzisen Zusammenfassung eine theoretische Diskussion, die sich auf hohem Reflexionsniveau bewegt und für die Politische Ökonomie durchaus zukunftsweisenden Charakter hat. Dies betrifft zwei der in der aktuellen Forschung wohl am intensivsten diskutierten Fragen, nämlich zum einen die eingangs skizzierte Komplementaritätsproblematik und zum anderen die Frage nach der Logik institutionellen Wandels.

Im Hinblick auf institutionelle Komplementarität hat die Untersuchung gezeigt, dass auch in erfolgreichen Produktionsregimen Widersprüche zwischen institutionellen Teilsphären auftreten 
können und diese weniger starke Kopplungen aufweisen als von Hall und Soskice suggeriert wird. Was die Theorie institutionellen Wandels anbetrifft, regt das Buch zur Thematisierung inkrementeller Veränderungsprozesse sowie zu einer Hinterfragung einseitig funktionalistischer Argumente an.

Unter dem Strich lässt sich festhalten, dass es sich bei der besprochenen Publikation um einen erkenntnisreichen und gut lesbaren Forschungsbeitrag handelt, der nicht nur dem Fachpublikum, sondern auch interessierten Lesern aus anderen Disziplinen uneingeschränkt empfohlen werden kann.

Philipp Klages

\section{POLITISCHE SOZIOLOGIE}

Thomas Bräuninger: Internationale Institutionenpolitik. Die Wahl von Entscheidungsregeln für die Meeresbodenbehörde. Frankfurt a.M./ New York: Campus 2002. 297 Seiten. ISBN 3-593-36631-2. Preis: € 34,90.

Bei der Arbeit von Thomas Bräuninger handelt es sich um eine Dissertation, die im Kontext der interdisziplinären Mannheimer DFG-Forschergruppe „Institutionalisierung Internationaler Verhandlungen" (1998-2005) entstanden ist. Der Autor untersucht das komplexe Verhandlungssystem der Dritten Seerechtskonferenz (Third United Nations Conference on the Law of the Sea, UNCLOS III), bei der 150 Staaten von 1973 bis 1982 verhandelten. Die daraus resultierende Seerechtskonvention unternahm eine umfassende Kodifizierung und Fortschreibung des Seerechts, u.a. für den Bereich der Schifffahrt, der Nutzungsrechte maritimer lebender und nichtlebender Ressourcen und des maritimen Umweltschutzes. $\mathrm{Zu}$ einem zentralen Thema entwickelte sich der Gegenstand der Nutzung der mineralischen Tiefseeressourcen und deren institutionelle Regelung im Rahmen einer neu einzurichtenden Internationalen Meeresbodenbehörde (International Seabed Authority). Eine Minderheit der führenden Industriestaaten, darunter die USA, die UdSSR und die meisten Mitgliedstaaten der EG stimmten 1982 gegen die Seerechtskonvention oder enthielten sich. Es handelte sich dabei um potenzielle Investoren, die am ehesten in der Lage waren, den lukrativen Abbau von Meeresbodenmineralien vorzunehmen. Diese befürworteten weiter das geltende konventionelle Recht des freien Zugangs zu den Tiefseeressourcen (Open
Access-Regime), um einer befürchteten Ex-postExpropriation riskanter und aufwendiger Investitionen bei der Explorierung und dem Abbau der Mineralienbestände durch die im Rahmen der Seerechtskonvention erwarteten Regulierung und Umverteilung zu entgehen. Die Nichtratifikation durch diese Staaten hätte folglich bedeutet, dass die Konvention faktisch ineffektiv geblieben wäre. Erst eine weitere Initiative Anfang der 90er führte zu einem allseitig akzeptierten Verhandlungskompromiss im Rahmen der so genannten Durchführungsvereinbarung (1994), die nunmehr den Industriestaaten institutionelle Vorteile einräumte, d.h. sie konnten u.a. nicht mehr von einer Mehrheit der Entwicklungsländer überstimmt werden. Die institutionellen Arrangements der Seerechtskonvention und der Durchführungsvereinbarung unterscheiden sich dabei u.a. durch die Einführung eines Vierkammersystems mit absoluten Vetorechten.

Bräuninger gelingt es nach den wichtigen Arbeiten von James K. Sebenius (Negotiating the Law of the Sea, Cambridge: Harvard University Press 1984) und Robert L. Friedheim (Negotiating the New Ocean Regime, Columbia: University. of South Carolina Press 1993), zur dritten Seerechtskonferenz eine eigene theoretische Perspektive vorzuschlagen und eine originelle Sekundäranalyse von Friedheims Datensatz durchzuführen. Nach den mittlerweile eingetretenen Sättigungserscheinungen des neo-institutionalistischen Programms der „strukturinduzierten Gleichgewichte" stellt er die fundamentalere Frage, wie es denn zu Präferenzen über Regeln, Institutionen und Verfassungen kommt.

In einem ersten Schritt legt der Autor im Hinblick auf dieses zentrale Thema zunächst die wirtschaftlichen Interessen der Staaten offen. Hierbei unterscheidet er zwischen Konsumentenund Produzenteninteressen. Bei letzteren hebt er v.a. auf die speziellen Interessen der Produzenten terrestrischer Mineralien ab, die bei einem verstärkten Meeresbodenabbau Preisverluste und Absatzprobleme erwarten mussten und damit potenziell offen waren für Koalitionen mit den unterentwickelten Staaten. Die für die jeweiligen Staaten abgeleiteten Interessen werden für drei ausgewählte so genannte inhaltliche Regelungsgegenstände, nämlich Produktionsmengen, Produktionsabgaben und Verteilung der Einnahmen als operationale Hypothesen formuliert. Der Autor erwartet u.a., dass, je wahrscheinlicher die Aufnahme des Tiefseebergbaus seitens eines Staates, gemessen über den Indikator der in Forschung und Entwicklung Beschäftigten sei, desto wahrscheinlicher werde der Staat für ein regulationsund abgabefreies Abbauregime eintreten (90). 